\title{
SHORT-TERM LOAD FORECASTING USING THE ADAPTIVE NETWORK-BASED FUZZY INFERENCE SYSTEM
}

\author{
WEN-YEAU CHANG
}

Department of Electrical Engineering, St. John's University, Taiwan

The short-term load forecasting method plays an important role for electric utility that can help the electric utility to improve reliability and security of electricity supply. This paper proposed an adaptive network-based fuzzy inference system (ANFIS) based method for the short-term load forecasting.

To evaluate the accuracy of the proposed ANFIS based forecasting method, the load data of a practical electrical power system was used. The accuracy of the proposed method was evaluated using two indices, namely the maximum absolute percentage error, and the mean absolute percentage error. The forecasting data are found to be in close agreement with the realistic data. The numerical simulation results show that the proposed approach may achieve quite satisfactory forecasting of load.

KEYWORDS: Short-term Load Forecasting, Adaptive Network-based Fuzzy Inference System, Maximum Absolute Percentage Error, Mean absolute Percentage Error
\end{abstract}

Received: Jun 20, 2017; Accepted: Jul 06, 2017; Published: Jul 12, 2017; Paper Id.: IJEEERAUG20173

\section{INTRODUCTION}

Short-term load forecasting plays an important role in many areas of the electric power management system. Short-term load forecasting is used for the unit commitment, maintenance scheduling, hydro-thermal coordination and economic dispatch. Unexpected variations of the load may increase operating risk for the electricity system, because of the unbalancing of power.

There have been some methods adopted in short-term load forecasting. These load forecasting methods can be divided into two types, which are time series methods and biological swarm intelligence methods. In time series methods group, there are the auto regressive model (Anguita, et al 2012), particle swarm optimization-based auto regressive model (Yu, et al 2014), auto regressive moving average model (Hafen, et al 2014), auto regressive integrated moving average model (Zhu, et al 2012), auto regressive integrated moving average model-adaptive network-based fuzzy inference system method (Suhartono, et al 2012) and auto regressive integrated moving average model-GARCH approach (Khuntia, et al 2016).

By means of the development of biological swarm intelligence technology, several biological intelligence methods have been developed for load forecasting. There are, the neural network method (Sulaiman, et al 2016), the grey model (Sabri, et al 2011), the support vector machine method (Gao, et al 2012), and the fuzzy logic model (Khosravi, et al 2012). Since the load forecasting problem is multi-dimensional and nonlinear, the biological intelligence methods can forecast the load with enough accuracy.

The aim of this paper is, to propose the ANFIS based short-term load forecasting method. The 
organization of this paper is: Section 2 discusses the principle of adaptive network-based fuzzy inference system. The ANFIS based short-term load forecasting method is presented in Section 3. Numerical simulation results are presented and discussed in Section 4 and the conclusion is described in Section 5.

\section{PRINCIPLE OF THE ADAPTIVE NETWORK-BASED FUZZY INFERENCE SYSTEM}

Fuzzy logic is based on the human been thinking modeling. Fuzzy systems set up the rule base by estimation the relation functions from logical input to output (Kwan and Cai, 1994). The neural network model is one of the powerful intelligence tools by mean of self-learning ability; neural network model can learn knowledge from training data. The neural network method prefers in nonlinear or complex problems. The neural network models have been widely applied in forecasting problem. Adaptive network-based fuzzy inference system can take advantages of both the fuzzy rule base thinking modeling of the fuzzy system and learning ability, from training data of the neural network (Chang and Miao, 2015).

The ANFIS model was proposed by Jang in 1993 (Jang, 1993), which is based on a special fuzzy inference structure. The ANFIS model's output is a particular linear combination function of the input signals (Zhou, et al 2011). In ANFIS model, the input data is converted to fuzzy data by mean of the particular membership function for each input data. The particular membership function can be arbitrary function, but it depends on the particular data set of the system (Rahman, et al 2013). In this paper, the membership function and fuzzy rule is obtained by using the historical load data of electric power systems.

Figure 1 shows the architecture of ANFIS model. There are five layers in ANFIS model, from left side to right side the first layer is the fuzzy layer, the second layer is the product layer, the third layer is the normalized layer, the fourth layer is the defuzzification layer, and the fifth layer is the output layer. The nodes in the same layer have similar function style.

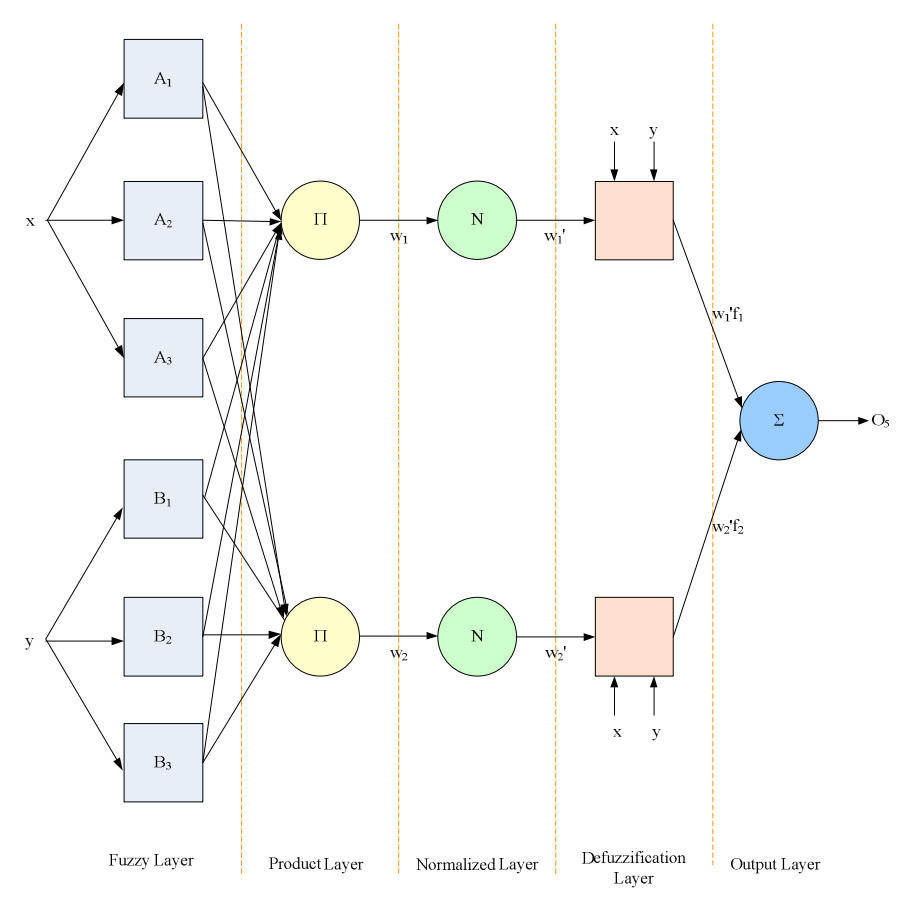

Figure 1: The Architecture of ANFIS Model 
Each layer's function of ANFIS can be described as following

- The adaptive nodes in the fuzzy layer will generate the membership levels of linguistic inputs. The fuzzy layer can use any kind of shape membership function such as the generalized bell functions. In fuzzy layer, $A_{1}, A_{2}, A_{3}, B_{1}$, $\mathrm{B}_{2}, \mathrm{~B}_{3}$ are each fuzzy set's linguistic labels to divide the membership functions for the adaptive nodes. The node's relationship function between the input and output is expressed as Eq. (1):

$$
O_{1, i}=\mu_{A i}(x), \quad i=1,2,3
$$

Where, $O_{1, i}$ is output of the node in the first layer, and $\mu_{A i}$ is the membership function of the $i$ th fuzzy set $\mathrm{A}_{i}, i=1,2,3$.

- The rule nodes in the product layer are designated as $\Pi$, which represents the shooting strength of each rule node. The output function is the product of the input signal of the node. The node's relationship function between the input and output is expressed as Eq.(2):

$O_{2, i}=w_{i}=\mu_{A i}(x) \times \mu_{B i}(y), \quad i=1,2,3$

Where, $O_{2, i}$ is output of the node in the second layer, $\mu_{A i}$ is the membership function of the $i$ th fuzzy set $\mathrm{A}_{i}, i=1,2,3$, and $\mu_{B i}$ is the membership function of the $i$ th fuzzy set $\mathrm{B}_{i}, I=1,2,3$.

- The fixed nodes in the normalized layer are labeled as $\mathrm{N}$. The output function is the normalize sum of the input signal of the node. The node's relationship function between the input and output is expressed as Eq.(3):

$$
O_{3, i}=w_{i}{ }^{\prime}=\frac{w_{i}}{w_{1}+w_{2}+w_{3}}, \quad i=1,2,3
$$

Where, $O_{3, i}$ is the output of the node in the third layer, $w_{e}$ is output of the node in the second layer.

- In the defuzzification layer, the adaptive nodes are the nodes, which are besides the nodes in the fuzzy layer. Based on specific consequent parameters, each adaptive node calculates the weighted sum of the rule outputs. The node's relationship function between the input and output is expressed as Eq. (4):

$O_{4, i}=w_{i}^{\prime} f_{i}=w_{i}{ }^{\prime}\left(p_{i} x+q_{i} y+r_{i}\right), \quad i=1,2,3$

where $O_{4, i}$ is output of node in the fourth layer, and $p_{i}, q_{i}, r_{i}$ are the specific consequent parameters of the node in defuzzification layer.

- The output layer is the last layer of ANFIS. The output node in this layer is labeled as $\Sigma$. The output node will calculates the weighted sum of the node input signals. The node's relationship function between the input and output is expressed as Eq. (5):

$$
O_{5, i}=\sum_{i=1}^{3} w_{i}^{\prime} f_{i}, \quad i=1,2,3
$$

(5)

Where, $O_{5, i}$ is the output of the node in this layer, $w_{i} f_{i}$ is output of the node in the fourth layer. 


\section{ANSIF BASED LOAD FORECASTING METHOD}

Figure 2 shows the structure of the proposed ANSIF based load forecasting model. In this paper, the structure of ANSIF model contains five layers. There are 4 nodes in the fuzzy layer in which $\mathrm{x}$ presents the actual load in 20 minutes ago, y presents the actual load in 10 minutes ago, z presents the actual current load, and u presents the temperature forecasting value for 10 minutes ahead. There is only one node in the output layer in which o presents the 10 minutes ahead load forecasting. The number of fuzzy rules in the ANSIF model is 81 . The generalized bell function is used for membership functions.

The processing steps of ANSIF based load forecasting method is briefly described in the following:

Step 1: Collect the load data and temperature data of electric power system to create the load database.

Step 2: Normalize all the data in load database.

Step 3: Prepare the training set from load database for the ANSIF.

Step 4: Using the two-pass process training scheme to train the load forecasting ANSIF model.

Step 5: Save the parameters of the trained ANFIS, as the training procedure is finished.

Step 6: Use the trained ANFIS to forecast the load of electric power system.

\section{NUMERICAL SIMULATION RESULTS}

To evaluate the accuracy of the proposed ANFIS based forecasting method, ANFIS based forecasting method has been applied for load forecasting by using the practical load data of the Taiwan electrical power system (Taipower system). The test load data is collected from the Taipower system every 10 minutes in Mid-May of 2015. Due to the difference of regional load characteristics, the load database is divided into 4 regions: northern Taiwan region, central Taiwan region, southern Taiwan region, and whole Taiwan region. The load forecasting testing results of weekday (Monday to Friday) for four regions are shown below. In the load forecasting testing, the every 10 minutes load data and temperature data of following days are selected: May 11-15, 2015. The load database has 864 sets of data, which were divided into training database for ANFIS, which were composed of 720 sets of data recorded from May 11-14 2015, and testing database, which were composed of 144 sets of data recorded from May 152015. 


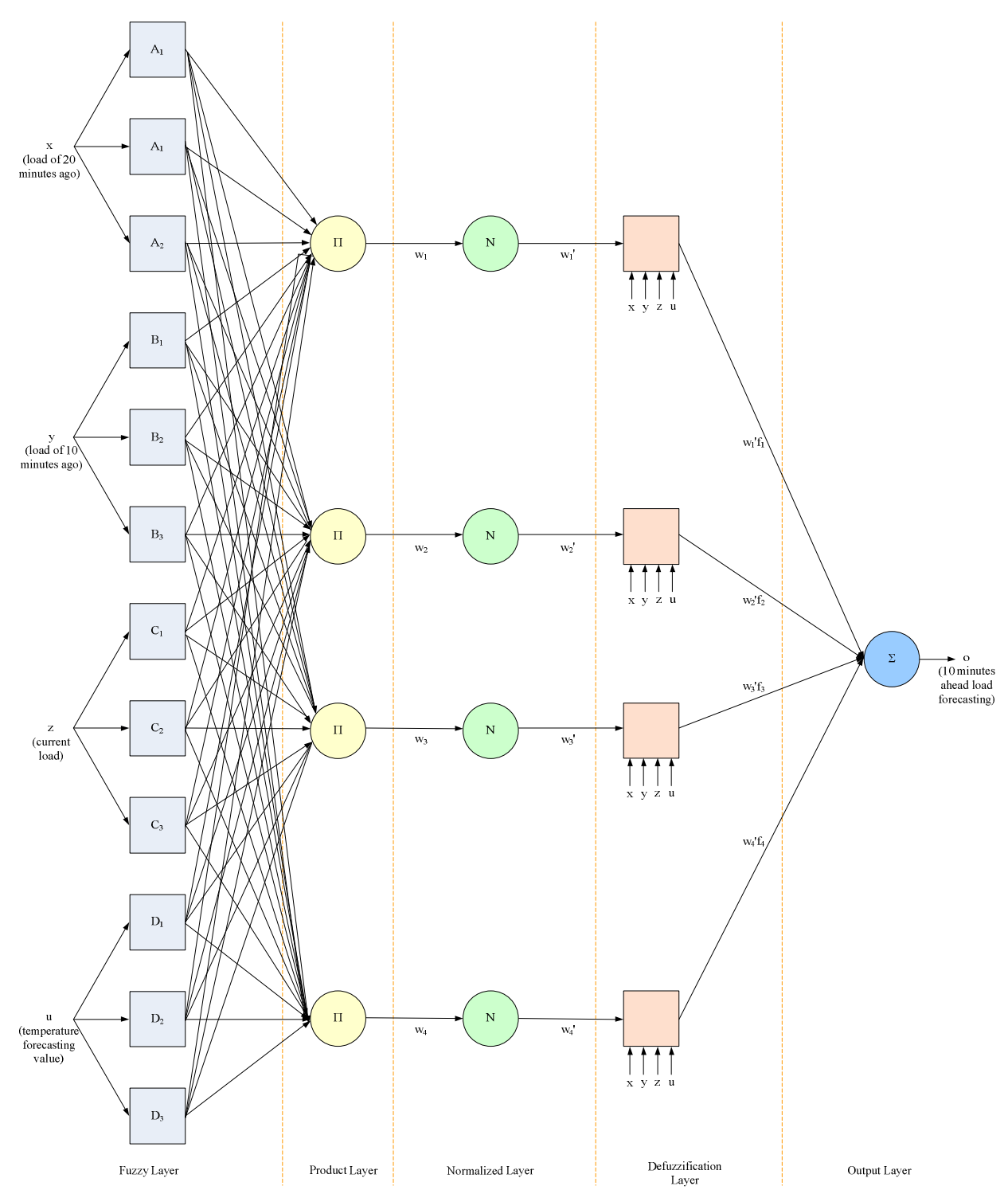

Figure 2: The Structure of the Proposed ANSIF Based Load Forecasting Model

The performance evaluation of the proposed forecasting method in this paper is evaluated by using two indices, which are the maximum absolute percentage error and the mean absolute percentage error. In the Load forecasting testing results for northern Taiwan region with the ANFIS based forecasting method, the maximum absolute percentage error is $3.832 \%$ and the mean absolute percentage error is $1.743 \%$. in the Load forecasting testing results for central Taiwan region, the maximum absolute percentage error is $4.012 \%$ and the mean absolute percentage error is $1.535 \%$. Load forecasting testing results for southern Taiwan region, the maximum absolute percentage error is $2.982 \%$ and the mean absolute percentage error is $1.355 \%$. In the Load forecasting testing results for whole Taiwan region, the maximum absolute percentage error is $3.756 \%$ and the mean absolute percentage error is $1.542 \%$. Table 1 shows the values of the criteria for evaluating the accuracy of the four regions for load forecasting. The comparing the four regions data, a general conclusion that may be drawn from the obtained results is that the ANSIF based load forecasting model can forecast the load accurately. 
Table 1: The Accuracy Evaluation of the Four Regions of Taipower System for Load Forecasting

\begin{tabular}{|l|c|c|}
\hline Region of Taipower system & $\begin{array}{c}\text { Maximum Absolute } \\
\text { Percentage Error }\end{array}$ & $\begin{array}{c}\text { Mean Absolute } \\
\text { Percentage Error }\end{array}$ \\
\hline Northern Taiwan region & $3.832 \%$ & $1.743 \%$ \\
\hline Central Taiwan region & $4.012 \%$ & $1.535 \%$ \\
\hline Southern Taiwan region & $2.982 \%$ & $1.355 \%$ \\
\hline Whole Taiwan region & $3.756 \%$ & $1.542 \%$ \\
\hline
\end{tabular}

\section{CONCLUSIONS}

Based on the ANFIS model, this paper has proposed a new method to forecast the load of electric power system. The accuracy and the effectiveness of the proposed approach have been verified by using numerical testing results. The testing results indicate that an accurate load forecasting can be achieved by using the ANFIS approach.

\section{ACKNOWLEDGMENTS}

The author would like to express his acknowledgements to the Ministry of Science and Technology of ROC for the financial support under Grant MOST 103-2632-E-129-002-MY3.

\section{REFERENCES}

1. Anguita, D., Ghelardoni, L, \& Ghio, A.(2012). Long-term energy load forecasting using auto-regressive and approximating support vector regression. Proceedings of the 2012 IEEE International Energy Conference and Exhibition, 842-847. doi: 10.1109/Energy Con. 2012.6348269

2. Chang, W. Y., \& Miao, H. C. (2015). Short-term solar power forecasting using the adaptive network-based fuzzy inference system. Advances in Engineering Research, 22, 640-643.

3. Gao, R., Zhang, L. \& Liu, X.(2012). Short-term load forecasting based on least square support vector machine combined with fuzzy control. Proceedings of the 2012 10th World Congress on Intelligent Control and Automation, 1048-1051. doi: 10.1109/ WCICA.2012.6358034

4. Hafen, R. P., Samaan, N., Makarov, Y. V., Diao, R. \& Lu, N. (2014). Joint seasonal ARMA approach for modeling of load forecast errors in planning studies. Proceedings of the 2014 IEEE PES T\&D Conference and Exposition, 1-5. doi: 10.1109/TDC.2014.6863150

5. Jang, J.S.R. (1993). ANFIS: adaptive-network-based fuzzy inference system. IEEE Transactions on Systems, Man and Cybernetics, 23(3), 665-685. doi: 10.1109/21.256541

6. Khosravi, A., Nahavandi, S., Creighton, D. \& Srinivasan, D.(2012). Interval type-2 fuzzy logic systems for load forecasting: a comparative study. IEEE Transactions on Power Systems, 27, 1274-1282. doi: 10.1109/TPWRS.2011.2181981

7. Khuntia, S.R., Rueda, J.L., \& van der Meijden, M.A.M.M.(2016). Volatility in electrical load forecasting for long-term horizon - An ARIMA-GARCH approach. Proceedings of the 2016 International Conference on Probabilistic Methods Applied to Power Systems, 1-6. doi: 10.1109/PMAPS.2016.7764184

8. Kwan, H.K., \& Cai, Y.(1994). A fuzzy neural network and its application to pattern recognition. IEEE Transactions on Fuzzy Systems, 2(3), 185-193. doi: 10.1109/91. 298447

9. Rahman, M., Islam, A.H.M.S., Nadvi, S.Y.M., \& Rahman, R.M.(2013). Comparative study of ANFIS and ARIMA model for weather forecasting in Dhaka. Proceedings of the 2013 International Conference on Informatics, Electronics \& Vision, 1-6. doi: 10.1109/ICIEV. 2013.6572587 
10. Sabri, Y., Hariyanto, N. \& Fitriana, F.(2011). Spatial short-term load forecasting using grey dynamic model specific in tropical area. Proceedings of the 2011 International Conference on Electrical Engineering and Informatics, 1-6. doi: 10.1109/ICEEI.2011. 6021776

11. Suhartono, Puspitasari, I., Sjahid Akbar, M. \& Lee, M.H.(2012). Two-level seasonal model based on hybrid ARIMA-ANFIS for forecasting short-term electricity load in Indonesia. Proceedings of the 2012 International Conference on Statistics in Science, Business and Engineering, 1-5. doi: 10.1109/ICSSBE.2012.6396642

12. Sulaiman, S.M., Aruna Jeyanthy, P., \& Devaraj, D.(2016). Artificial neural network based day ahead load forecasting using smart meter data. Proceedings of the 2016 Biennial International Conference on Power and Energy Systems: Towards Sustainable Energy, 1-6. doi: 10.1109/PESTSE.2016.7516422

13. Yu, J., Lee, H., Jeong, Y. \& Kim, S.(2014). Short-term hourly load forecasting using PSO-based AR model. Proceedings of the 15th International Symposium on Soft Computing and Intelligent Systems, 1449-1453. doi: 10.1109/SCIS-ISIS.2014.7044868

14. Zhou, H., Wu, X.H., \& Li, X.G.(2011). An ANFIS model of electricity price forecasting based on subtractive clustering. Proceedings of the 2011 IEEE Power and Energy Society General Meeting, 1-5. doi: 10.1109/PES.2011.6039228

15. Zhu, X. and Shen, M.(2012). Based on the ARIMA model with grey theory for short term load forecasting model. Proceedings of the 2012 International Conference on Systems and Informatics, 564-567. doi: 10.1109/ICSAI.2012.6223060 
